\title{
Sistema de monitoreo y comunicación en tiempo real mediante una interfaz de usuario, para mejorar el proceso de estibación y el registro del producto embotellado en la industria Pascual Tizayuca.
}

\author{
Monitoring and real-time communication system through a user interface to improve the \\ stowage process and the registry of the bottled product in Pascual Tizayuca's factory.
}

\author{
Eddher Cruz-Segura ${ }^{a}$, Osmar Santillan-Ramírez ${ }^{b}$, Luis R. Coello-Galindo ${ }^{c}$
}

\begin{abstract}
:
This work deals the development of an interface for real time monitoring control variables that are immersed in the process of product stacking, within the Pasqual Company such as: the position and speed of the industrial forklift, likewise the registration of the product that is being worked. The interface programming is carried out using Visual Studio and is complemented by applying the ESP32 microcontroller for system communication with the forklift operator using Bluetooth technology. Similarly, some electronic components will be used; such as phototransistors for product counting, color sensors for type product recognition handled, the $F C$ 03 optical encoder for monitoring the industrial forklift speed.

In order to monitor the actions of the system in real time, a graphical user interface will be implemented to visualize the speed of the forklift actuators, the registration and control of the product carried out daily to optimize the working time in the plant, to have a better control of the variables that get involved in the product stacking process and provide reliable real-time information.
\end{abstract}

\section{Keywords:}

Graphic interface, Monitoring, Real Time, ESP32

\section{Resumen:}

En éste trabajo se presenta el desarrollo de una interfaz para monitorear en tiempo real algunas variables que intervienen en el proceso de estibación de productos dentro de la industria Pascual, como lo son: la posición del montacargas, la velocidad del mismo y el registro del producto con el que se está trabajando. La interfaz se lleva a cabo utilizando Visual Basic y se complementa aplicando el microcontrolador ESP32 para la comunicación del sistema con el montacarguista empleando la tecnología Bluetooth; y algunos componentes electrónico tales como un fototransistor para el conteo del producto, sensores de colores para el reconocimiento del tipo del producto manejado, el encoder óptico FC-03 para monitorear la velocidad del montacargas.

Para poder monitorear en tiempo real las acciones del sistema, se implementará una interfaz gráfica de usuario para visualizar la velocidad de los actuadores del montacargas, el registro y control del producto realizado diariamente para poder optimizar el tiempo de trabajo en la planta, tener un mejor control de las variables que intervienen en el proceso de estibación de productos y brindar información en tiempo real confiable.

\section{Palabras Clave:}

Interfaz gráfica, Monitoreo, Tiempo Real, ESP32.

\section{Introducción}

Un sistema de comunicaciones es un conjunto de dispositivos que son utilizados con la finalidad de transmitir, emitir y recibir señales de todo tipo, como voz, datos, audio, video, etc., además dichas señales pueden ser del tipo digital o analógico.

Un sistema de comunicaciones puede describirse fácilmente mediante tres elementos básicos; un transmisor, el cual se encarga de generar la señal que se

a Escuela Superior de Tizayuca, Universidad Autónoma del Estado de Hidalgo, México, Km. 2.5. Carretera Federal Tizayuca - Pachuca, 43802, Tizayuca, Hidalgo. E-mail: cr333169@uaeh.edu.mx

${ }^{\mathrm{b}}$ Escuela Superior de Tizayuca, Universidad Autónoma del Estado de Hidalgo, México, Km. 2.5. Carretera Federal Tizayuca - Pachuca, 43802, Tizayuca, Hidalgo. E-mail: sa334948@uaeh.edu.mx

${ }^{\mathrm{c}}$ Escuela Superior de Tizayuca, Universidad Autónoma del Estado de Hidalgo, México, Km. 2.5. Carretera Federal Tizayuca - Pachuca, 43802, Tizayuca, Hidalgo. E-mail:

coello@uaeh.edu.mx 
desea y acoplarla de tal forma que pueda viajar a través del canal, mediante procedimientos como modulación, filtrado, codificación, etc.; un medio de transmisión, el cual será el canal mediante el cual la señal va a viajar, y puede ser desde fibras ópticas, cables coaxiales, hasta el mismo aire; y finalmente un receptor, que realiza el procedimiento inverso del transmisor con la finalidad de reconstruir la señal y que ésta sea lo más parecida a la original (Franco, 2019).

Mientras que el monitoreo:

- Es un proceso continuo.

- Implica la recolección de datos en múltiples momentos a lo largo del ciclo de ejecución del programa, incluso al principio para establecer una línea base.

- Se puede utilizar para determinar si es necesario realizar ajustes a las actividades durante la intervención para mejorar los resultados esperados (Nina, 2009).

Las variables a las que se les da lectura y visualización como se presenta a continuación son extraídas de una problemática real basada en un caso de estudio en la Industria Pascual en donde se tuvo la oportunidad de extraer información que ha permitido, diseñar y desarrollar el sistema que ayude a mejorar dichas problemáticas, tales como la pérdida de tiempo en el proceso de estibación, el registro del producto que aún en su totalidad no es automático, y no tienen un sistema de monitoreo y comunicación, por lo antes mencionado el proyecto está basado en Pascual y se adapta a lo observado dentro de ésta.

\section{Descripción del sistema.}

La interfaz se desarrolló en su totalidad en el entorno de desarrollo integrado de Visual Studio 2015, bajo el lenguaje de programación de Visual Basic, permitiendo una conexión con el microcontrolador ESP32 para leer los datos de los sensores y del encoder óptico, así como también una conexión a una base de datos para llevar un control del registro del producto con el que se esté trabajando (Javier, 1990).
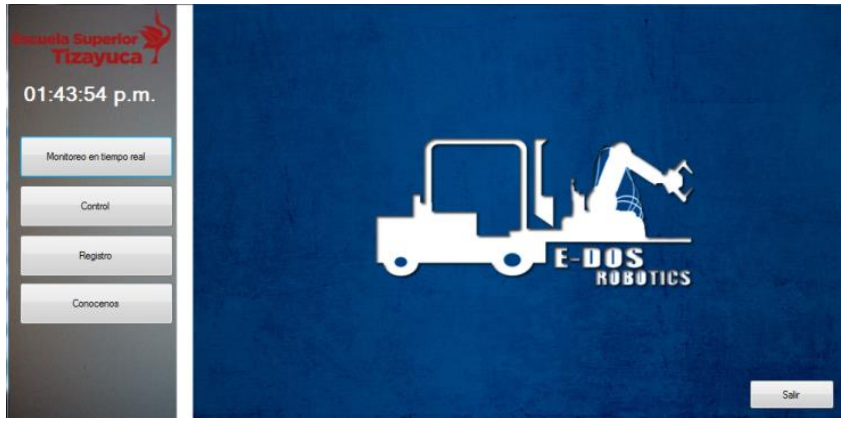

Figura 1: Ventana principal de la interfaz.

Para la realización de la interfaz de usuario se tomaron en cuenta algunas problemáticas que tienen en la industria Pascual de Tizayuca por lo que se consideró tener opciones de acuerdo a las mismas. De las cuales las principales fueron:

1. El registro a mano del producto que se tiene en almacén para su posterior captura en la base de datos

2. Carencia de un sistema de monitoreo y comunicación para conocer la posición de los montacargas y en consecuencia les genera pérdida de tiempo.

A continuación se presentan las distintas ventanas que posee la interfaz, así como de la descripción de sus funciones.

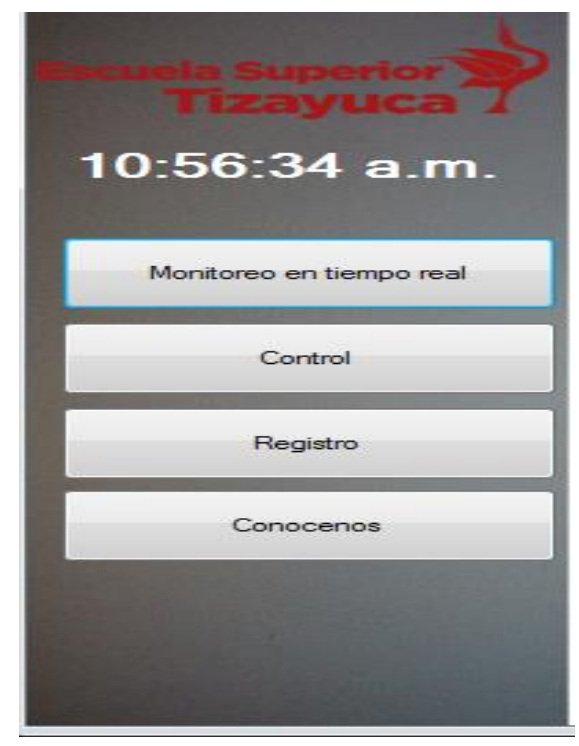

Figura 2: Botones de las opciones de la interfaz.

Como se muestra en la figura 2, la interfaz posee algunas opciones que aportan solución y control a las variables que influyen en el mal funcionamiento dentro del proceso de estibación del producto, como se habla de tiempo real el sistema (interfaz gráfica), tiene la funcionalidad de sincronización con el reloj en donde se esté ejecutando, incluyendo los segundos, por lo que en el momento en que 
se ejecuta el usuario tiene visualización de la hora. Posterior a esto se observan cuatro botones los cuales son:

- Monitoreo en tiempo real.

- Control.

- Registro.

- Conócenos.

En la figura 3 se muestra la ventana de monitoreo en tiempo real y en este momento está habilitada para trabajar con dos dispositivos, el croquis está diseñado en base a la industria Pascual de Tizayuca y representada con un software de diseño llamado Sketchup.

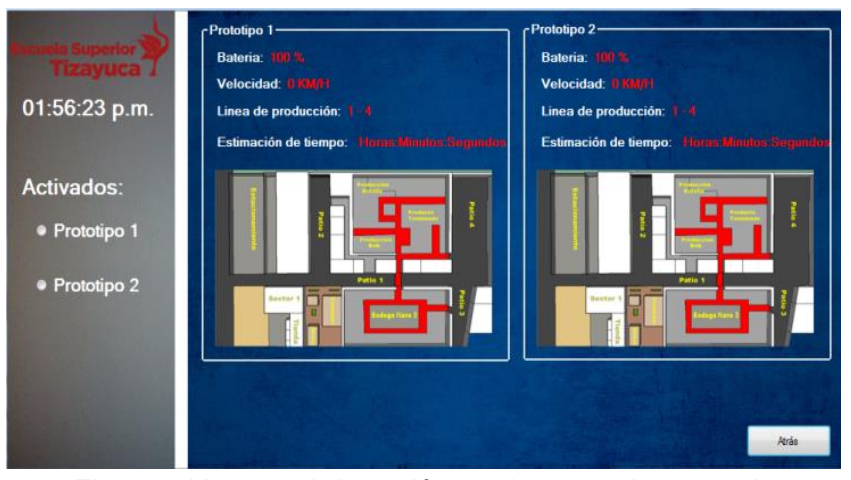

Figura 3: Ventana de la opción monitoreo en tiempo real.

En la figura 3.1 se muestra el monitoreo en tiempo real de donde está ubicado el montacargas de acuerdo al plano a escala del área de estibación del producto, de igual forma se muestran algunos indicadores relacionados al monitoreo como son: batería, velocidad, línea de producción por donde se está desplazando el montacargas y el tiempo de estimación que se refiere la cantidad de tiempo en que ha estado en operación el montacargas.

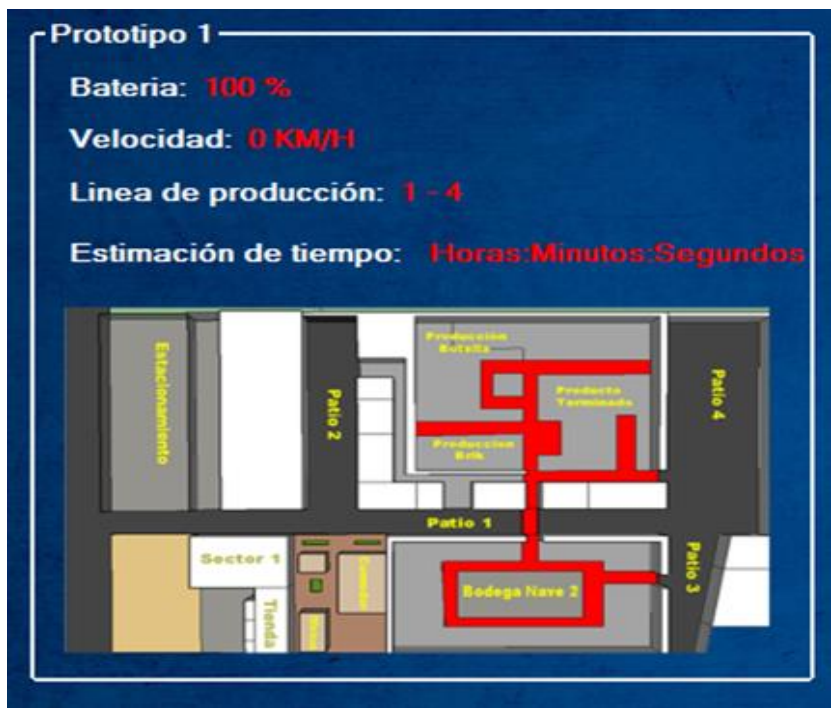

Figura 3.1 Monitoreo del prototipo 1.
En la figura 4 se muestra el estado de los dispositivos conectados a la interfaz si está conectado o desconectado, así mismo se puede dar la opción de conectar con el dispositivo y las opciones de posibles dispositivos para conectar. Por último viene el indicador de velocidad en rpm de los actuadores del montacargas.
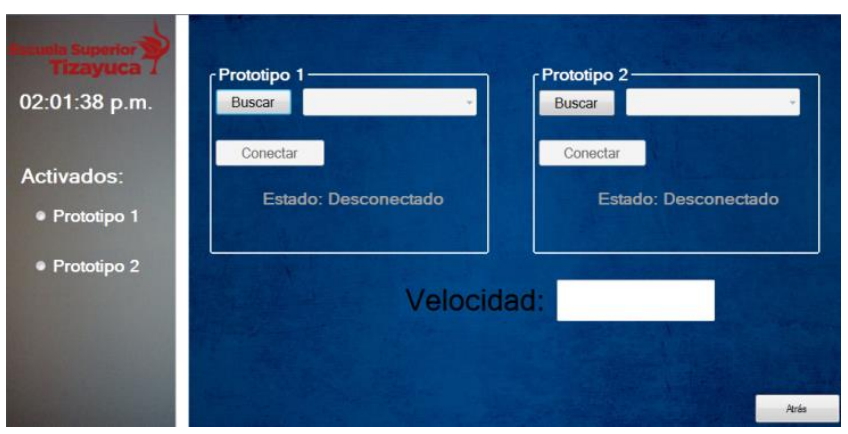

Figura 4: Ventana de la opción control.

En la figura 5 se muestra la información de los desarrolladores de la interfaz como recurso de alguna posible duda en cuestión de la operatividad, funcionamiento o falla.

Cabe mencionar que la interfaz aún se encuentra en etapa de desarrollo ya que se le pudieran aumentar más ventanas y aún falta la implementación de algunas funciones.
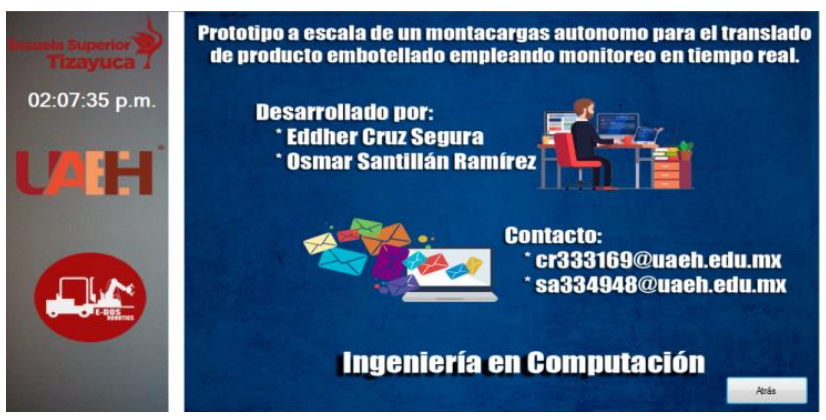

Figura 5: Ventana de la opción conócenos.

\section{Análisis de la interfaz.}

Para analizar la interfaz de usuario se ocupó un diagrama de navegación con el cual, ha permitido tener un panorama más explícito de cómo es la transición de ventana a ventana y cómo el usuario interactuaría con la misma, ya que posee varios botones y funciones que pudieran ser un poco confuso si es la primera vez que se ocupa. 
Inclusive este diagrama puede servir para que el operador de la interfaz tenga una idea de cómo ésta va funcionando dependiendo de qué se desee hacer.

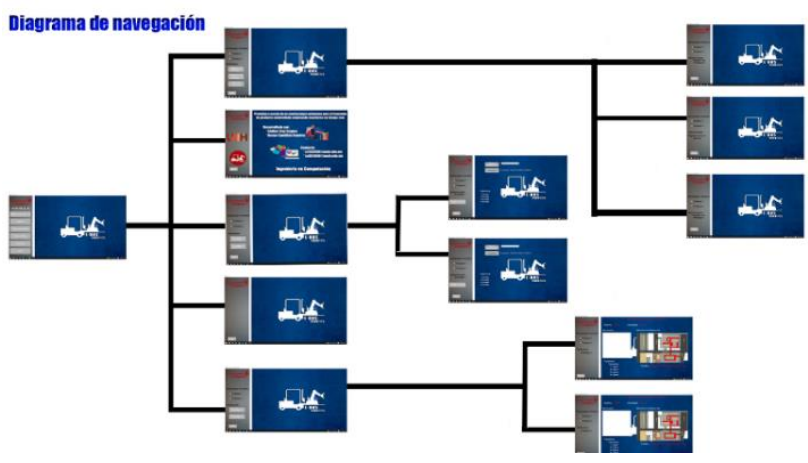

Figura 6: Diagrama de navegación del sistema.

\section{Descripción de los elementos de hardware}

Para el hardware se ocupó en primera instancia el microcontrolador ESP32 que envía a la interfaz el dato leído por el encoder, este dispositivo tiene muchas características que lo hacen de gran utilidad, entre las principales se pueden resaltar las siguientes (Luis, 2018):

- Módulo wifi y módulo bluetooth.

- RAM de 520kB.

- Frecuencia de reloj de $240 \mathrm{MHz}$.

- CPU Dual Core Tensilica LX6.

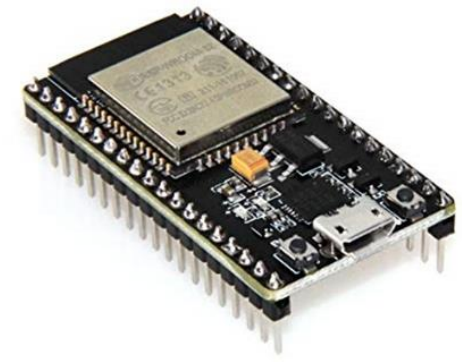

Figura 7: Módulo microcontroladorESP32.

De igual manera se ocupó un encoder óptico para poder leer las revoluciones a las que gira el actuador. Esto se hace mediante un emisor-transmisor que emite y recibe una señal de alta frecuencia, además posee un disco con ranuras en donde al girar el actuador se van produciendo interrupciones, el programa contabiliza la cantidad de interrupciones que genera el encoder y se genera el resultado de las r.p.m. a las que el motor está girando (Francisco. M, 2019).

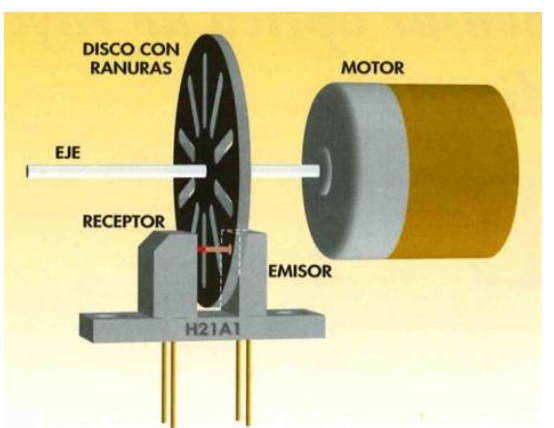

Figura 8: Encoder óptico.

Finalmente el sistema queda interactuando para conocer las revoluciones del actuador de la siguiente manera: el encoder entrega el conteo de interrupciones, el ESP32 recibe el dato y lo envía a la interfaz gráfica, ésta lo procesa y lo visualiza gráficamente.

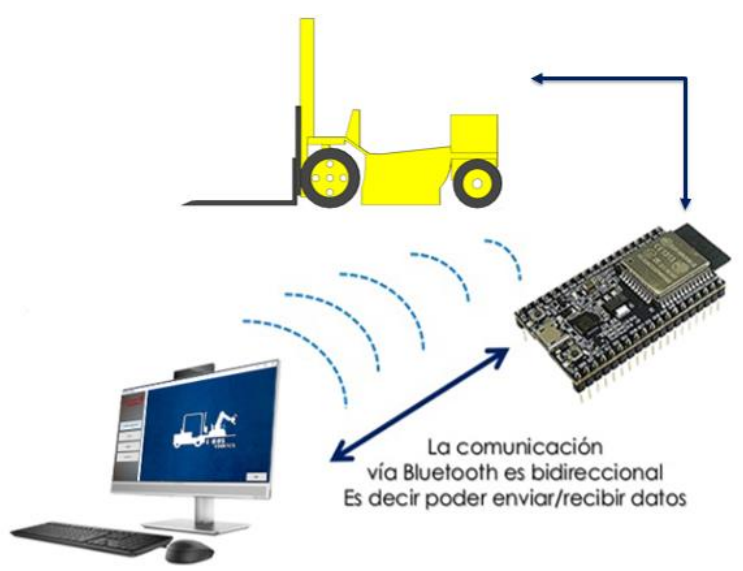

Figura 9: Diagrama de funcionalidad del sistema.

\section{Demostración del avance de la funcionalidad de la interfaz}

En la figura 10 se muestra el comportamiento de la interfaz cuando se conecta con el ESP32 y así mismo se demuestra la funcionalidad de la misma, en esa figura se puede observar que cuando la interfaz detecta el dispositivo ESP32 se activa la casilla de prototipo 1, en la opción buscar aparece el puerto al que está conectado y de igual forma tiene un submenú desplegable para seleccionar el puerto al que se desea conectar en caso de que tuviéramos otro dispositivo conectado, la etiqueta de desconectado pasa a un estado de conectado y se marca en negritas para resaltar el estado en que se encuentra.

Inmediatamente aparecen las revoluciones a las que se está desplazando los actuadores del montacargas; así 
mismo un botón para desconectar el prototipo en el momento que se desee dejar de trabajar.

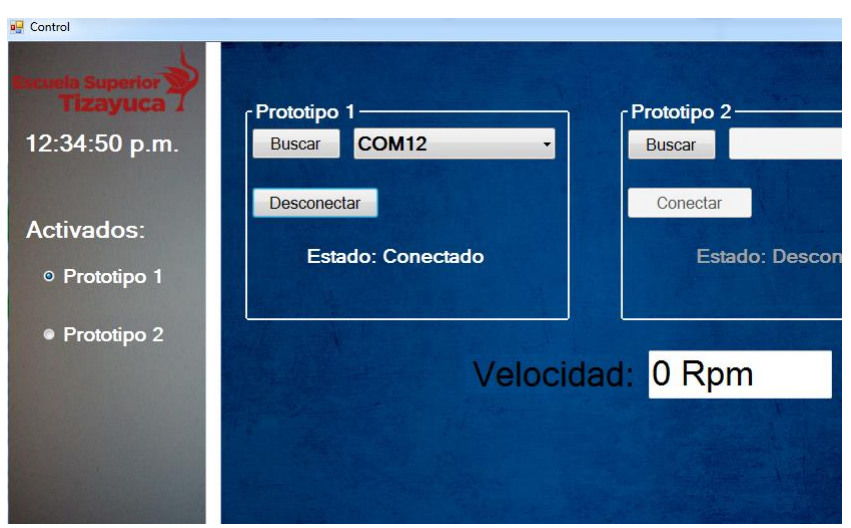

Figura 10: Pantalla de control.

En la figura 11 se muestra una lectura que brinda el Encoder de las revoluciones a la que está girando el actuador, el dato mostrado es de suma importancia, porque dentro de la industria Pascual se tiene un límite de velocidad dentro de sus instalaciones esto con el fin de evitar accidentes. Así que se ha planeado el monitoreo de la velocidad del montacargas para que el operador del sistema sepa si está operando dentro del rango de velocidad adecuado y en caso de no ser así pueda notificársele con el fin de evitar un posible accidente.

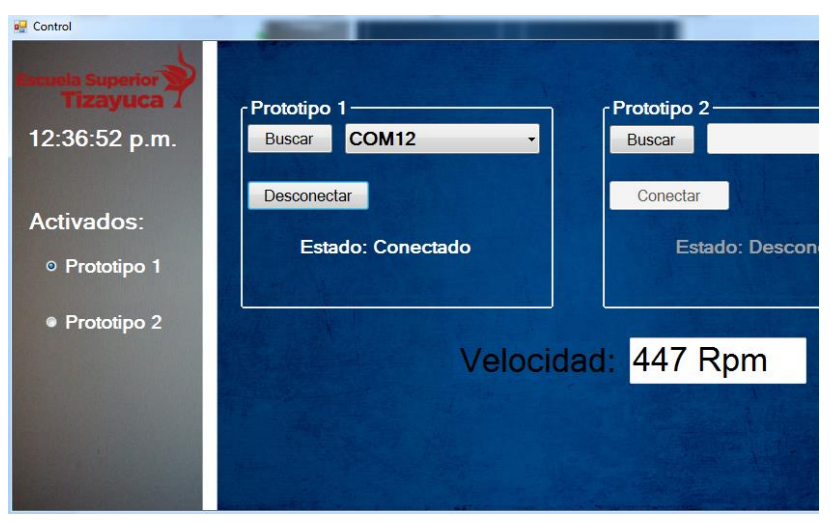

Figura 11: Pantalla de control.

\section{Análisis y discusión de resultados}

Al analizar los resultados obtenidos al momento el enfoque del sistema de monitoreo (interfaz gráfica), cumple con el propósito de visualización de las variables que se pretenden mostrar. En primera instancia se está trabajando con las r.p.m. para conocer la velocidad a la que desplaza el móvil, se evalúa que el dispositivo ESP32 funciona de manera correcta y se sincroniza de manera eficiente con la interfaz gráfica desarrollada en Visual Studio.

El encoder logra censar la velocidad generada por el motor, funcionando como el lector primario de dicha variable, ya que la interfaz gráfica es la que interpreta y muestra el dato para su visualización en tiempo real, se puede afirmar que la combinación del microcontrolador ESP32, el encoder y la interfaz gráfica pueden generar, interpretar y emitir la variable de velocidad en r.p.m.

Hablando del diseño de la interfaz gráfica, que será el sistema de monitoreo, se basan en las siguientes características (Alba B, 2014).

1. Sencillez, claridad y concisión.

2. Familiaridad y contextualidad.

3. Interactividad o "feedback".

4. Importancia de las funciones estándar.

5. Posición y colores de las acciones.

Teniendo esta referencia se afirma que la interfaz posee estos elementos dentro de su diseño, es apta para el usuario y logrando mostrar hasta éste momento la variable de velocidad de manera exitosa; es como se llega a la discusión y afirmación de que el sistema de monitoreo hasta este momento es funcional y logra emitir la variable deseada.

Hablando de conectividad del microcontrolador ESP32 con la interfaz de usuario, se puede decir que se ha comprobado que conecten mediante Wifi y Bluetooth; sin embargo, se estará ocupando la tecnología Bluetooth. Hasta este momento se ha probado conectar dos módulos ESP32 a la interfaz sin generar algún problema de error de conexión con la interfaz, posteriormente se estará trabajando con el monitoreo en tiempo real.

Pero hasta el momento los resultados generados son los correctos y permite seguir adelante con las demás funciones que tendrá el sistema.

Cabe mencionar que el proyecto tiene un impacto directo con las problemáticas de la industria Pascual. La variable trabajada (rpm) y las que se estarán trabajando (monitoreo en tiempo real y comunicación entre el montacarguista y el operador de la interfaz) tienen el objetivo de mejorar, comunicar y monitorear el proceso de estibación, impacta de manera muy específica en dicho proceso ya que brinda información confiable, precisa y en tiempo real por lo que se vuelve un sistema completo.

\section{Conclusiones}

Para lograr un sistema que pueda llevar acabo monitoreo y comunicación en tiempo real, se debe saber qué se tiene que aplicar tanto software como hardware, ya que van de la mano y son los pilares de este proyecto; sin embargo, para crear un sistema de este tipo se debe tener en cuenta qué tecnología se va a ocupar, cómo se va aplicar y con qué fin, es decir que se tenga la aplicación que se le dará para solucionar el problema.

Un sistema de monitoreo y comunicación conlleva a tener un objetivo claro de qué variables se van a trabajar y cómo se les va a dar seguimiento y de qué forma serán procesadas, para tener un control y una visualización de las mismas, éste proyecto está basado en un caso de estudio de la industria Pascual con variables reales que 
generan un retraso en el sistema de producción de dicha industria.

La interfaz gráfica ayuda logró ayudar al procesamiento de la información recibida de los elementos de hardware del sistema, así mismo permitió visualizar los resultados de manera sencilla y eficiente tanto para los operadores de los montacargas como a los encargados de las diferentes áreas de producción. La versatilidad del mismo permite poder integrársele en un futuro más funciones en base a las necesidades o requerimientos que sean solicitados. Algo a destacar sería que permitiría combinar con distintos elementos de hardware que le puedan brindar mayor robustez y operatividad para alcanzar los objetivos en cada área; es por esto que para éste proyecto se le da un peso mayoritario a la interfaz y se ocupa como sistema de monitoreo y comunicación.

El sistema presentado está desarrollado pensando en la solución o mejoramiento del proceso de estibación de la industria pascual; son soluciones propuestas por los desarrolladores del proyecto y se resalta que, siendo un sistema modificable, se podría adaptar a las necesidades de otras industrias, empresas, compañías etc. ya que la tecnología y las herramientas que al momento están en el mercado ayuda y se pueden implementar para ofrecer un sistema adaptado a las necesidades del mercado.

\section{Referencias}

[1] Franco G. (2019). Sistemas de comunicaciones. Repositorio digital de la Facultad de Ingeniería - UNAM Sitio web: http://www.ptolomeo.unam.mx:8080/xmlui/bitstream/handle/1 32.248.52.100/861/A6.pdf?sequence $=6$

[2] Nina Frankel, Anastasia Gage. (2009). Fundamentos de monitoreo y evaluación. PEPFAR, pp. 7.

[3] García de Jalón, J. I. Rodríguez, A. Brazález (1990). Aprenda Visual Basic 6.0 como si estuviera en primero. Universidad de Navarra Nafarroako Unibertsitatea.

[4] Luis, LL (2018). ESP32, el "hermano mayor" del esp8266 con wifi y bluetooth. Recuperado el 01 de enero de 2019 de Ingeniería, informática y diseño. Sitio web: https://www.luisllamas.es/esp32/

[5] Francisco, M (2017). Encoder ¿cómo funciona? y sus tipos. 05 de enero de 2019 de Ingeniería Mecafenix. Sitio web: https://www.ingmecafenix.com/automatizacion/encoder/

[6] Alba, B (2014). Cinco pautas para diseñar una buena interfaz de usuario. 14 de octubre de 2018 de Baquia hablando de Tecnología desde $1999 . \quad$ Sitio web: https://www.baquia.com/emprendedores/2014-02-17-cincopautas-para-disenar-una-buena-interfaz-de-usuario-1 